\title{
A semi-staggered dilation-free finite volume method for the numerical solution of viscoelastic fluid flows on all-hexahedral elements
}

\author{
Mehmet SAHIN \\ FSTI-IE-LMF, Swiss Federal Institute of Technology, \\ CH-1015 Lausanne, Switzerland ${ }^{1}$ \\ Helen J. WILSON \\ Department of Mathematics, UCL \\ Gower Street, London WC1E 6BT, UK
}

\begin{abstract}
The dilation-free semi-staggered finite volume method presented in Int. J. Numer. Meth. Fluids 49 (2005) 959-974 has been extended for the numerical solution of viscoelastic fluid flows on allquadrilateral(2D)/hexahedral(3D) meshes. The velocity components are defined at element node points, while the pressure term and the extra stress tensor are defined at element centroids. The continuity equation is satisfied exactly within each element. An upwind least square method is employed for the calculation of the extra stresses at control volume faces in order to maintain stability for hyperbolic constitutive equations. The time stepping algorithm used decouples the calculation of the extra stresses from the evaluation of the velocity and pressure fields by solving a generalised Stokes problem. The resulting linear systems are solved using the GMRES method provided by the PETSc library with an ILU $(\mathrm{k})$ preconditioner obtained from the HYPRE library. We apply the method to both two and three dimensional flow of an Oldroyd-B fluid past a confined circular cylinder in a channel with blockage ratio 0.5 .
\end{abstract}

Keywords: Viscoelastic Fluid Flow; Finite Volume; Unstructured Methods; Three Dimensional; Iterative Methods

\section{INTRODUCTION}

Over the past decades, significant progress has been achieved in the numerical solution of viscoelastic fluid flows. Nevertheless, viscoelastic flow simulations still remain a challenging task in term of accuracy, stability, convergence and required computer power. In this work we attempt to tackle these issues by extending our semi-staggered dilation-free finite volume method [27] to viscoelastic fluid flows.

Recently, finite volume methods have been widely used for viscoelastic fluid flows. Yoo and Na [32] solved planar contraction flow of an Oldroyd-B fluid on a non-uniform staggered grid using the SIMPLER algorithm. Sasmal [28] presented an upwind finite volume algorithm based on the stream functionvorticity approach in the Elastic Viscous Split Stress (EVSS) form with fully staggered flow variables to solve the UCM model in an axisymmetric contraction flow. Dou and Phan-Thien [8] implemented an unstructured finite volume method based on the SIMPLER algorithm with the EVSS formulation for a simplified PTT constitutive model on triangular meshes with collocated flow variables along with an equal order interpolation. Oliveira et al. [22] developed a collocated finite volume method on nonorthogonal grids; the velocity-stress-pressure decoupling was removed by using an interpolation similar to that of Rhie and Chow [24]. Mompean and Deville [20] and Xue et al. [31] used a fully staggered finite volume method to simulate three dimensional planar contraction flow. Wapperom and Webster [34] used a second-order hybrid scheme which uses a finite element method for the mass-momentum balance equations and a finite volume method for the hyperbolic stress equations. In the present paper we employ a semi-staggered finite volume method on all-hexahedral elements. The main advantage of this

\footnotetext{
${ }^{1}$ Current address: Department of Mathematics, UCL, Gower Street, London WC1E 6BT, UK
} 
semi-staggered approach is that it has a better coupling compared to the collocated approach while being able to treat complex configurations unlike the fully staggered approach. Furthermore, the summation of the continuity equation within each element can be exactly reduced to the domain boundary, which is important for global mass conservation. But the most appealing feature of the method is that it leads to a very simple algorithm consistent with the boundary and initial conditions required by the viscoelastic fluid flow equations.

In earlier finite volume works (Yoo and $\mathrm{Na}$ [32] and Sasmal [28]) first-order upwind approximations were used for the convective terms of the stress constitutive equation, which tends to cause severe numerical diffusion whenever the flow is not aligned with the grid orientation. The use of higher-order approximations was reported by Mompean and Deville [20], Oliveira et al. [22] and Alves et al. [1]. Mompean and Deville [20] used a quadratic upstream interpolation (QUICK) scheme [18] to study the flow of the Oldroyd-B fluid in 3D domains. Oliveira et al. [22] applied a second-order linear upwind differencing scheme to two-dimensional Poiseuille entry flow and the flow around a confined cylinder for the UCM fluid. In a later study, Alves et al. [1] implemented high resolution interpolation schemes MINMOD and SMART in order to improve stability and accuracy. In the present work a least square upwind interpolation scheme is employed. Although it is widely used for the computation of turbulent flows $[4,2]$ as far as we are aware this is the first time it has been used for viscoelastic fluid flow calculations. As indicated by Anderson and Bonhaus [2], the use of a least square procedure for evaluating the gradient term in order to extrapolate variables to the boundaries of the control volumes is far superior to the use of gradients calculated with Green's theorem on highly stretched meshes. In addition, this least square procedure for evaluating the gradient term does not require the construction of a dual control volume, which is rather difficult in $3 \mathrm{D}$.

As in the work of Caola et al. [7], we use a time-splitting technique which decouples solution of a generalised Stokes problem from the calculation of the extra stresses. This is used to step the timedependent equations in time until a steady state is reached. Although this decoupling limits the allowable time step, both steps can be solved efficiently by using preconditioned Krylov subspace methods. A preconditioned generalised minimum residual (GMRES) method [26] was first applied to steady state viscoelastic fluid flow calculations by Fortin and Fortin [12] for calculation of the stick-slip problem. Baaijens [3] devised a block preconditioner with GMRES and applied it to the DEVSS/DG discretisation for axisymmetric contraction flow of a UCM fluid using a fully coupled Newton's method. However, these iterative solvers for steady state viscoelastic flow calculations suffer from poor robustness and large memory requirements as the size of the problem increases. Therefore they are unsuitable for 3D simulations. On the other hand, high resolution 2D numerical results have been presented in the literature using time-dependent simulations. Caola et al. [7] presented numerical results for up to 751,110 degrees of freedom for Oldroyd-B flow past a confined cylinder. Recently, Kim et al. [17] have presented numerical results for up to 1,362,480 degrees of freedom using an adaptive incomplete LU (AILU) preconditioner with variable reordering.

In our problem there is a zero block resulting from the divergence-free constraint. We use an upper triangular right preconditioner which results in a scaled discrete Laplacian instead of a zero block in the original system, which is more physical than the AILU preconditioner. Unfortunately, this leads to a significant increase in the number of non-zero elements following the matrix-matrix multiplication. However, the new system may be efficiently preconditioned using incomplete LU (ILU) preconditioner. The implementation of the preconditioned Krylov subspace algorithm was carried out using the PETSc [5] software package developed at Sandia National Laboratories. The preconditioning uses the ILU(k) preconditioner [15] provided by the HYPRE library [10], a high performance preconditioning package developed at Lawrence Livermore National Laboratory, which we access through the PETSc library. The use of these highly efficient libraries allows us to present numerical results for up to 2,961,143 degrees of freedom on a single processor for the 3D Oldroyd-B flow past a confined cylinder. This is even larger than the previously reported $2 \mathrm{D}$ results in the literature, which require less memory due to smaller matrix bandwidth in $2 \mathrm{D}$.

The method presented here is tested by solving the classical benchmark problem of the flow past a confined circular cylinder in a channel. In both two and three dimensional calculations, the blockage ratio is set to 0.5. In three dimensional calculations the spanwise aspect ratio is set to 2.5. In the literature, the problem of viscoelastic flow past a confined circular cylinder has been studied by many researchers $[1,7,8,11,14,17,23]$ and to date all numerical simulations of this problem have been limited to two 
dimensions. The current paper represents, as far as we are aware, the first numerical 3D viscoelastic fluid flow calculations for a confined circular cylinder in a channel. These full 3D numerical simulations are necessary in order to capture the true nature of the flow patterns. In this paper we present numerical results up to a Weissenberg number of 1.2. However, both 2D and 3D results failed to converge at higher Weissenberg numbers due to the classical high Weissenberg number problem (HWNP), and the converged $2 \mathrm{D}$ numerical results beyond $W e=0.7$ indicate that the solutions are far from mesh convergence in a small region in the wake of the cylinder. Although the recent log conformation of Hulsen et al. [14] seems to remove the convergence problem, the authors reported that they could not find any sign of convergence for stress in the wake beyond some rather small Weissenberg number (order 1). In our 3D calculations at moderately large Weissenberg numbers we observe the emergence of corner vortices at the wall-cylinder junction. In addition, the distance between the streamtraces downstream of the cylinder is no longer uniform close to the side walls. These observations are in accord with the experimental results of Shiang et al. [29] and McKinley et al. [19].

The paper is structured as follows: In Section 2 we describe our semi-staggered finite volume method with the iterative method for the solution of the resulting system of algebraic equations. In Section 3 the proposed method is applied to the well-known problem of the 2D/3D flow of Oldroyd-B fluid past a circular cylinder in a channel. The numerical results are presented for a sequence of viscoelastic calculations with increasing Weissenberg number. Conclusions are presented in Section 4.

\section{MATHEMATICAL and NUMERICAL FORMULATION}

The governing equations for three dimensional unsteady flow of an incompressible and isothermal OldroydB fluid can be written in dimensionless form as follows: the continuity equation

$$
-\nabla \cdot \mathbf{u}=0
$$

the momentum equations

$$
R e\left[\frac{\partial \mathbf{u}}{\partial t}+(\mathbf{u} \cdot \nabla) \mathbf{u}\right]+\nabla p=\beta \nabla^{2} \mathbf{u}+\nabla \cdot \mathbf{T}
$$

and the constitutive equation for the Oldroyd-B model

$$
W e\left[\frac{\partial \mathbf{T}}{\partial t}+(\mathbf{u} \cdot \nabla) \mathbf{T}-(\nabla \mathbf{u})^{\top} \cdot \mathbf{T}-\mathbf{T} \cdot \nabla \mathbf{u}\right]=(1-\beta)\left(\nabla \mathbf{u}+\nabla \mathbf{u}^{\top}\right)-\mathbf{T}
$$

In these equations $\mathbf{u}$ represents the velocity vector, $p$ is the pressure and $\mathbf{T}$ is the extra stress tensor. The dimensionless parameters are the Reynolds number $R e$, the Weissenberg number $W e$ and the viscosity ratio $\beta$. Integrating the differential equations (1) and (3) over a quadrilateral $(2 \mathrm{D}) /$ hexahedral $(3 \mathrm{D})$ element $\Omega_{e}$ with boundary $\partial \Omega_{e}$ gives

$$
-\oint_{\partial \Omega_{e}} \mathbf{n} \cdot \mathbf{u} \mathrm{d} S=0
$$

$$
\begin{aligned}
W e\left[\int_{\Omega_{e}}\left(\frac{\partial \mathbf{T}}{\partial t}-(\nabla \mathbf{u})^{\top} \cdot \mathbf{T}-\mathbf{T} \cdot \nabla \mathbf{u}\right) \mathrm{d} V+\oint_{\partial \Omega_{e}}(\mathbf{n} \cdot \mathbf{u}) \mathbf{T} \mathrm{d} S\right] & =(1-\beta) \oint_{\partial \Omega_{e}}[\mathbf{u n}+\mathbf{n u}] \mathrm{d} S-\int_{\Omega_{e}} \mathbf{T} \mathrm{d} V
\end{aligned}
$$

and integrating the differential equation (2) over an arbitrary irregular dual control volume $\Omega_{d}$ with boundary $\partial \Omega_{d}$ gives

$$
R e\left[\int_{\Omega_{d}} \frac{\partial \mathbf{u}}{\partial t} \mathrm{~d} V+\oint_{\partial \Omega_{d}}(\mathbf{n} \cdot \mathbf{u}) \mathbf{u} \mathrm{d} S\right]+\oint_{\partial \Omega_{d}} p \mathbf{n} \mathrm{d} S-\beta \oint_{\partial \Omega_{d}} \mathbf{n} \cdot \nabla \mathbf{u} \mathrm{d} S-\oint_{\partial \Omega_{d}} \mathbf{n} \cdot \mathbf{T} \mathrm{d} S=0
$$

Here $\mathbf{n}$ represents the outward normal unit vector. For the remainder of this section we restrict ourselves to the discretisation of $2 \mathrm{D}$ flows; the extension to $3 \mathrm{D}$ is straightforward. Fig. 1 illustrates typical four node quadrilateral elements with a dual finite volume constructed by connecting the centroids $c_{i}$ of the 
elements which share a common vertex. The discrete contribution computed by connecting the element centroids $c_{1}$ and $c_{2}$ for the momentum equation is given by

$$
\begin{array}{r}
\operatorname{Re}\left[\frac{\mathbf{u}_{P}^{n+1}+\mathbf{u}_{1}^{n+1}+\mathbf{u}_{2}^{n+1}}{3 \Delta t}-\frac{\mathbf{u}_{P}^{n}+\mathbf{u}_{1}^{n}+\mathbf{u}_{2}^{n}}{3 \Delta t}\right] V_{P 12}+R e\left[\mathbf{n}_{12} \cdot\left(\frac{\mathbf{u}_{1}^{n}+\mathbf{u}_{2}^{n}}{2}\right)\right]\left(\frac{\mathbf{u}_{1}^{n+1}+\mathbf{u}_{2}^{n+1}}{2}\right) S_{12} \\
\quad+\mathbf{n}_{12}\left(\frac{p_{1}^{n+1}+p_{2}^{n+1}}{2}\right) S_{12}-\beta \mathbf{n}_{12} \cdot\left(\frac{\nabla \mathbf{u}_{1}^{n+1}+\nabla \mathbf{u}_{2}^{n+1}}{2}\right) S_{12}-\mathbf{n}_{12} \cdot\left(\frac{\mathbf{T}_{1}^{n+1}+\mathbf{T}_{2}^{n+1}}{2}\right) S_{12}
\end{array}
$$

where $V_{P 12}$ is the area between the points $P, c_{1}$ and $c_{2}$ and $S_{12}$ is the length between the points $c_{1}$ and $c_{2}$. The other contributions are calculated in a similar way. The velocity vector at the element centroids $c_{i}$ is computed from the element vertex values using simple averages and the gradient of velocity components $\nabla \mathbf{u}$ are calculated from Green's Theorem:

$$
\nabla \mathbf{u}_{i}=\frac{1}{V} \oint_{\partial \Omega_{e}} \mathbf{n u} \mathrm{d} S
$$

where the line integral on the right-hand side of equation (8) is evaluated using the mid-point rule on each of the element faces. The continuity equation is integrated in a similar manner within each element. The constitutive equation is integrated within each element assuming that the extra stresses $\mathbf{T}_{i}$ and velocity gradients $\nabla \mathbf{u}_{i}$ are constant:

$$
\begin{aligned}
W e\left[\frac{\mathbf{T}_{i}^{n+1}-\mathbf{T}_{i}^{n}}{\Delta t} V+\sum_{f=1}^{4}\left(\mathbf{n} \cdot \mathbf{u}^{n}\right) \mathbf{T}_{f}^{n+1} S-\left(\nabla \mathbf{u}_{i}^{n}\right)^{\top} \cdot \mathbf{T}_{i}^{n+1} V\right. & \left.-\mathbf{T}_{i}^{n+1} \cdot \nabla \mathbf{u}_{i}^{n} V\right] \\
& =(1-\beta)\left(\nabla \mathbf{u}_{i}^{n}+\left(\nabla \mathbf{u}_{i}^{n}\right)^{\top}\right) V-\mathbf{T}_{i}^{n} V .
\end{aligned}
$$

where $\mathbf{T}_{f}$ is the value of the extra stress at the segment/face centres of the quadrilateral/hexahedral elements. In equation (9) the right hand side relates the Newtonian viscous stress to the extra stress tensor and therefore they should be evaluated at the same time level. In order to extrapolate the extra stresses to the boundaries of the finite volume elements a second-order upwind least square interpolation is used. Any component of the extra stress tensor $\phi$ may be extrapolated to the boundaries of the finite volume elements using a Taylor series expansion about the cell centres:

$$
\phi_{\text {face }}=\phi_{\text {cell }}+\nabla \phi \cdot \mathbf{r}
$$

where $\nabla \phi$ represents the gradient of extra stress components at the cell centres and $\mathbf{r}$ is the vector extending from the cell centre to the control volume face centres. A least square procedure is used to compute $\nabla \phi$ using the neighbouring element cell centre values. For example, each neighbouring cell centre value may be expressed as

$$
\phi_{i}=\phi_{0}+\phi_{x}\left(x_{i}-x_{0}\right)+\phi_{y}\left(y_{i}-y_{0}\right),
$$

This leads to

$$
\left[\begin{array}{cc}
x_{1}-x_{0} & y_{1}-y_{0} \\
x_{2}-x_{0} & y_{2}-y_{0} \\
\vdots & \vdots \\
x_{N}-x_{0} & y_{N}-y_{0}
\end{array}\right]\left[\begin{array}{c}
\phi_{x} \\
\phi_{y}
\end{array}\right]=\left[\begin{array}{c}
\phi_{1}-\phi_{0} \\
\phi_{2}-\phi_{0} \\
\vdots \\
\phi_{N}-\phi_{0}
\end{array}\right]
$$

This overdetermined system of linear equations may be solved in a least square sense using the normal equation approach, in which both sides are multiplied by the transpose. The modified system is solved using $Q R$ factorisation provided by the Intel Math Kernel Library in order to avoid the numerical difficulties associated with solving linear systems with near rank deficiency for highly stretched meshes. The use of this least square approximation for the gradient term in computing the convective term results in the same coefficients as computed from a second-order linear upwind interpolation on uniform Cartesian meshes. Therefore, our approximation is second-order (like the second-order linear upwind interpolation). 
The time-dependent finite volume discretisation of the above equations leads to a linear system of equations of the form

$$
\left[\begin{array}{ccc}
A_{\tau \tau} & A_{\tau u} & 0 \\
A_{u \tau} & A_{u u} & A_{u p} \\
0 & A_{p u} & 0
\end{array}\right]\left[\begin{array}{l}
\tau \\
u \\
p
\end{array}\right]=\left[\begin{array}{c}
b_{1} \\
b_{2} \\
0
\end{array}\right]
$$

which needs to be solved for the new flow variables (at time level $n+1$ ) at each time step. Although the system matrix of (13) is indefinite due to the zero diagonal block resulting from the divergence-free constraint, recent results indicate that this system is similar to that arising in saddle point problems $[6,25]$, and that the indefiniteness of the problem does not represent a particular difficulty. We did not consider the EVSS formulation in this paper due to the significant increase in the number of unknowns in $3 \mathrm{D}$.

In practice, the solution of equation (13) does not converge very quickly and it is rather difficult to construct robust preconditioners for the whole coupled system. Therefore, we decouple the system by using a time-splitting technique which decouples the calculation of extra stresses from the evaluation of the velocity and pressure fields by solving a generalised Stokes problem. However, due to the zero diagonal block resulting from the divergence-free constraint, an ILU(k) type preconditioner cannot be used directly for the saddle point problem. Here, we consider an upper triangular right preconditioner in order to avoid problems arising from the zero block. The modified system becomes

$$
\left[\begin{array}{cc}
A_{u u} & A_{u p} \\
A_{p u} & 0
\end{array}\right]\left[\begin{array}{cc}
I & -A_{u p} \\
0 & I
\end{array}\right]\left[\begin{array}{l}
q_{1} \\
q_{2}
\end{array}\right]=\left[\begin{array}{cc}
A_{u u} & A_{u p}-A_{u u} A_{u p} \\
A_{p u} & -A_{p u} A_{u p}
\end{array}\right]\left[\begin{array}{c}
q_{1} \\
q_{2}
\end{array}\right]=\left[\begin{array}{c}
b_{2}-A_{u \tau} \tau \\
0
\end{array}\right] .
$$

and the zero block is replaced with $A_{p u} A_{u p}$, which is a scaled discrete Laplacian. Unfortunately, this leads to a significant increase in the number of non-zero elements due to the matrix-matrix multiplication. However, the new system may be solved efficiently by using preconditioned Krylov subspace methods. The implementation of the preconditioned Krylov subspace algorithm and the matrix-matrix multiplications were carried out using the PETSc [5] software package developed at Sandia National Laboratories. Although there are several Krylov subspace algorithms readily available in the PETSc library, we only employ the GMRES algorithm [26] for the problems presented in this paper, due to its stability. The Krylov subspace dimension is set to 100 for all cases. The preconditioning uses the ILU(k) preconditioner [15] provided by the HYPRE library [10], a high performance preconditioning package developed at Lawrence Livermore National Laboratory, which we access through the PETSc library. In the current calculations we could afford to use ILU(4) or above in the 2D cases, but for the 3D cases we could only afford to use ILU(0) due to the large memory requirement in $3 \mathrm{D}$. The block preconditioners given in $[9,3,27]$ are not considered here because of the significant increase in the number of inner iterations for convergence at the problem sizes given here. In addition, we did not use the filtering matrix of [27] because when we use right preconditioner for equations (14) and set the relative residual to $10^{-8}$ or lower, we observe the disappearance of pressure oscillations even for problems with a singular pressure field such as the lid-driven cavity problem of [27].

\section{NUMERICAL EXPERIMENTS}

In this section the proposed method is applied to the well-known $2 \mathrm{D} / 3 \mathrm{D}$ flow past a circular cylinder in a channel. The numerical results presented here are obtained using Euler implicit time stepping as given in Section 2, on a single Itanium2 1.3Ghz/3MB cache processor available on an SGI Altix 3700 parallel machine.

\subsection{Two dimensional numerical results}

The problem of two-dimensional viscoelastic flow past a confined circular cylinder is an attractive benchmark problem and has been studied by many researchers $[1,7,8,11,14,17,23]$. For this flow we consider a circular cylinder of radius $R$ positioned symmetrically between two parallel plates separated by a distance $2 H$. The blockage ratio $R / H$ is set to 0.5 and the lengths of the regions upstream and downstream of the cylinder are chosen to be $12 R$. The dimensionless parameters are the Reynolds number $R e=\langle v\rangle R / \eta$, the Weissenberg number $W e=\lambda\langle v\rangle / R$ and the viscosity ratio $\beta=\eta_{s} / \eta$. The physical parameters are the 
density $\rho$, the average velocity $\langle v\rangle$, the relaxation time $\lambda$, the zero-shear-rate viscosity of the fluid $\eta$ and the solvent viscosity $\eta_{s}$. The viscosity ratio $\beta$ is chosen to be 0.59 throughout this paper, which is the value used in the benchmarks for the Oldroyd-B fluid. In this work, fully developed velocity boundary conditions are imposed at the inlet boundary and natural (traction-free) boundary conditions are imposed at the outlet boundary. No-slip boundary conditions are imposed on all solid walls. The extra stresses are computed everywhere within the computational domain and their boundary conditions are introduced through their fluxes, using the analytical values at the inlet boundary.

In the present work three different meshes are employed: coarse mesh M1 with 6031 node points and 5824 elements, medium mesh M2 with 21735 node points and 21320 elements, and fine mesh M3 with 70312 node points and 69519 elements. Each of meshes M2 and M3 is generated by doubling the number of mesh points on the cylinder from the previous one. As may be seen in Fig. 2, the mesh is highly stretched on the cylinder surface, on the walls and in the wake behind the cylinder in order to resolve very strong stress gradients. The details of the mesh characteristics are given in Table I. In order to validate our code, this flow of an Oldroyd-B fluid past a confined circular cylinder is solved on meshes M1 to M3 for several different Weissenberg numbers, and contours of the extra stress component $T_{x x}$ and pressure are presented in Fig. 3 and Fig. 4 at $W e=0.7$. Even though we have an equal order interpolation for both the velocity and pressure, the pressure field is remarkable smooth and free from checkerboard pressure oscillations. The mesh convergence of the stress component $T_{x x}$ on the cylinder surface and along the centre line in the wake is presented in Fig. 5 and the extreme values of $T_{x x}$ on the cylinder surface and along the centre line in the wake are provided in Table II as a function of Weissenberg number. As seen in Fig. 5, mesh convergence is obvious at $W e=0.6$ and the mesh convergence trend is observed at $W e=0.7$. However there is no sign of mesh converge at higher Weissenberg numbers. In addition, the configuration tensor is no longer positive definite at $W e=0.9$. In the literature there are also large discrepancies for the stresses in the wake region; some researchers have suggested that there may be some numerical artifacts beyond $W e=0.7$. In Fig. 6 the stress component $T_{x x}$ on the cylinder surface and along the centre line in the wake are compared with the results of Fan et al [11] and Alves et al. [1] at a Weissenberg number of 0.7. These extreme values of $T_{x x}$ are compared with the other results available in the literature in Table III. The results of Fan et al. [11] were obtained using a Galerkin/least-squares $h p$ finite element method; Alves et al. [1] used a collocated high resolution finite volume method. The comparison shows good agreement bearing in mind the fact that the tangential mesh spacing on the cylinder surface behind the cylinder is approximately 5 times larger than the value used in the work of [1]. Therefore, we could expect even better agreement with further mesh refinement in the cylinder wake. In addition, a second-order linear upwind interpolation scheme for the convection term is implemented in order to compare its accuracy with the current least square interpolation scheme. The comparison is given in Fig. 7 for the benchmark problem of the Oldroyd-B fluid past a confined cylinder at $W e=0.7$. The computed results are indistinguable from one another. This should be expected since the use of this least square approximation for the gradient term in computing the convective term results the same coefficients as computed from the linear upwind interpolation on uniform Cartesian meshes. On coarser meshes the least square approximation gives smoother results than the linear upwind interpolation scheme. Although the convergence of the drag coefficient with mesh refinement is not considered to be a very good indicator of accuracy, the value for the steady state drag coefficients are tabulated in Table IV and compared with several other results available in the literature in Fig. 8. The present results indicate good agreement particularly with the results of Hulsen et al. [14], Fan et al. [11], Alves et al. [1] and Kim et al. [17]. All the present calculations are started from Stokes flow and stepped in time with a time step of 0.005 until all RMS values drop less than $10^{-8}$. The calculations up to a Weissenberg number of 0.8 converge monotonically to steady state. However, the calculations at $W e=0.9$ show oscillations in the RMS values during convergence to steady state. We believe that these oscillations are related to non-physical oscillations in $T_{x x}$ as seen in Fig. 5 at $W e=0.9$. Although Oliveira and Miranda [21] have recently showed a small time-periodic separation bubble behind the cylinder at a Deborah number of 1.3 for the FENE-CR model with $L^{2}=144$, we did not observe any similar flow structures for the Oldroyd-B fluid; our calculations beyond a Weissenberg number of 0.9 did not converge to any steady or time-periodic state on any of the meshes. 


\subsection{Three dimensional numerical results}

Full 3D numerical simulations are carried out for the flow of an Oldroyd-B fluid past a confined circular cylinder in a rectangular channel of depth $2 W$. The aspect ratio of the channel cross section is chosen to be $W / H=2.5$, which is taken computationally as large as possible in order to reduce the side wall effects and ensure that the flow within the channel is approximately 2D. The dimensionless parameters, Reynolds number and Weissenberg number, are defined based on the cylinder radius $R$ and the average velocity in the channel symmetry plane $\langle v\rangle$ as in the $2 \mathrm{D}$ case, rather than a volumetric average. This will allow us a better comparison between $2 \mathrm{D}$ and $3 \mathrm{D}$ results at the channel symmetry plane, since both flows have the same inflow velocity profiles. Should the volumetric mass flow in the channel be required, it can be calculated from the inflow velocity profile imposed at the inflow boundary:

$$
u(y, z)=1.6136 \sum_{k=1,3,5, \ldots}^{\infty}(-1)^{(k-1) / 2}\left[1-\frac{\cosh (k \pi z / 2 H)}{\cosh (k \pi W / 2 H)}\right]\left[\frac{\cos (k \pi y / 2 H)}{k^{3}}\right] ; \quad v(y, z)=w(y, z)=0
$$

The maximum inlet velocity is 1.5 and the average volumetric mass flow is 0.7798 . At the outflow the boundary conditions are set to zero derivative:

$$
\frac{\partial u}{\partial x}=\frac{\partial v}{\partial x}=\frac{\partial w}{\partial x}=0
$$

The extra stresses are computed within each element as in 2D and their boundary conditions are introduced through the analytical values for their fluxes at the inflow boundary.

In Fig. 9 we give a coarse computational mesh for these 3D simulations, which is created by sweeping the two dimensional cross-section of the coarse mesh M1 in the third dimension with 51 nonuniform node points. These $2 \mathrm{D}$ planes are highly stretched close to the side walls. The present calculations are carried out within the whole mesh without any use of symmetry since nonsymmetric 3D solutions may exist in viscoelastic fluid flows and these solutions can be extremely sensitive to geometric imperfections [30]. By introducing a small asymmetry into our mesh we hope to perturb these flows with infinitesimal asymmetric disturbances. A sequence of viscoelastic flow simulations is carried out for the flow of an Oldroyd-B fluid past a circular cylinder in a rectangular channel, with increasing Weissenberg number. As elastic effects in the flow become increasingly important, we observe a significant shift from the fore/aft symmetry of the equivalent creeping flow of a Newtonian fluid. In Fig. 10 the change in the velocity field may be seen from the velocity profiles in the $z=0$ plane at $x=0, x= \pm 1.5 R$ and $x= \pm 3 R$ at Weissenberg numbers of $0.0,0.7$ and 1.2. The flow near the junction with the side wall, both upstream and downstream of the cylinder, is highly three dimensional and there is a significant spanwise velocity component even for creeping flow of a Newtonian fluid at $W e=0.0$. As the Weissenberg number is increased these three dimensional effects become more predominant in the flow. The distance between the streamtraces is no longer uniform in the wake of the cylinder and they become more parallel to the side plates just downstream of the cylinder. Even though the flow upstream of the cylinder is not significantly changed at these low Weissenberg numbers, we observe a slight change in the streamtrace direction, being toward the centre of the channel rather than the side walls. These observations are in accord with the experimental results of Shiang et al. [29]. In addition, the 3D velocity profiles shown in Fig. 10 are very similar to velocity profiles measured by Verhelst and Nieuwstadt [33]. Although we cannot make a one-to-one comparison due to the different aspect ratio $(W / H=8)$, viscosity ratio $(\beta=0.73)$ and Deborah number $(D e=1.42)$ used in their experiment, it is particularly remarkable that a local maximum in the $u$-velocity profile at the centre line $(y=0)$ is captured at $W e=1.2$ as in Fig. 19 of [33] at $x=1.5 R$, just behind the cylinder. This is not seen in the two-dimensional numerical simulations of Oliveira and Miranda [21] with the FENE-CR model. The three dimensional effects may also be seen from the computed contour surfaces of stress component $T_{x x}$ and isobaric surfaces in Fig. 11 and Fig. 12 , respectively. The computed contour surfaces of $T_{x x}$ show that the cylinder wake is gradually extended downstream with increasing Weissenberg number. As the wake region develops behind the cylinder along the channel symmetry line the fluids within the viscoelastic boundary layer next to the cylinder surface move slightly slower, leading to higher extreme values of velocity outside the boundary layer, as may be seen from Fig. 10. However, this also leads to a decrease in $T_{x x}$ on the cylinder surface following the initial increase during startup. From the isobaric surfaces, a significant spanwise pressure gradient is observed 
along the $x= \pm R$ line on the cylinder surface, particularly close to the end-wall junctions. The pressure difference between $\pm 10 R$ on the channel centre line indicates a pressure drop of $52.44,49.40$ and 50.08 at Weissenberg numbers of 0.0, 0.7 and 1.2, respectively. This trend is similar to what has been established in the confined circular cylinder drag coefficient with Weissenberg number. In Fig. 13 we compare the extreme values of the stress component $T_{x x}$ on the cylinder surface and along the centre line in the wake with the two dimensional calculations on the same mesh M1. The comparison shows a $24.58 \%$ reduction in the extreme value of $T_{x x}$ on the cylinder surface and a $43.66 \%$ reduction in the extreme value along the channel centre line in the wake. As one of referees pointed out the main reason for this difference is the definition of Weissenberg number based on average velocity in the channel symmetry plane, rather than a volumetric average, which would in this case have given a value of $W e=0.55$ rather than $W e=0.7$. We also observed good agreement for $T_{x x}$ contours between our two-dimensional simulations at $W e=0.9$ and three-dimensional simulations at $W e=1.2$ where both flows have very close average mass flow rates.

Although the calculations at lower Weissenberg numbers converged monotonically and all the RMS values dropped to less than $10^{-4}$, the calculation at $W e=2.0$ diverged after several months of computation, due to the HWNP. As time goes to infinity the wake region region behind the cylinder gets thinner and thinner and the position of the extreme value of $T_{x x}$ approaches the cylinder surface. This high gradient region leads to oscillations in extra stress tensor (as in Fig. 5 at $W e=0.9$ ) and eventually leads to divergence of the solution, similar to what is observed in 2D. However, during this initial monotonic convergence (before any oscillations are seen in the extra stress tensor) we observe several interesting flow phenomena which are entirely absent at the lower Weissenberg numbers. From the streamtraces presented in Fig. 14 in the planes $z= \pm 4.99 R$, we observe the emergence of a corner vortex on the upstream side of the wall-cylinder junction, as in the experimental work of Shiang et al. [29]. In addition, the streamtraces start to merge close to the downstream wall-cylinder junction as shown in Fig. 15. This may possibly be the mechanism of a three dimensional instability such as that characterised in the previous investigations of McKinley et al. [19] and Shiang et al. [29]. However, we have not observed a three dimensional cellular structure within the flow. We believe that the instabilities observed in experiment are related to periodic oscillations in the $w$-velocity confined to a very small region just behind the cylinder, causing the merging of streamtraces. Additionally, the high curvature of the streamtraces in the third dimension, along the centre plane in the wake, may be relevant to three-dimensional viscoelastic instabilities.

More work is necessary, including the use of more refined meshes before any conclusion can be made on three dimensional instabilities in viscoelastic flow past a cylinder in a channel.

\section{CONCLUSIONS}

We have presented a new unstructured semi-staggered dilation-free finite volume method for the solution of viscoelastic fluid flow calculations on all-hexahedral elements. The time stepping algorithm used decouples the solution of the hyperbolic constitutive equation from the solution of the generalised Stokes problem. The use of the highly efficient PETSc and HYPRE libraries allows us to solve the 3D viscoelastic fluid flow around a confined circular cylinder on a single processor. However, the numerical results at high Weissenberg numbers did not converge due to the classical HWNP. The present simulations provide significant information about the 3D structure within the flow. Our numerical results indicate that the computed velocity and extra stresses are significantly different from those of $2 \mathrm{D}$ simulations, even on the vertical symmetry plane. In future work we will introduce adaptive local mesh refinement to refine the mesh only where necessary, allowing very accurate solutions at minimum cost, in order to study mesh convergence in the wake of the cylinder.

\section{ACKNOWLEDGMENTS}

This work was supported in part by EPSRC Grant GR/T11807/01. The authors acknowledge the use of UCL Research Computing facilities (Altix) and the EPFL Pleiades Linux Cluster. 


\section{References}

[1] M. A. Alves, F. T. Pinho and P. J. Oliveira, The flow of viscoelastic fluids past a cylinder: finitevolume high-resolution methods. J. Non-Newtonian Fluid Mech. 97, (2001), 207-232.

[2] W. K. Anderson and D. L. Bonhaus, An implicit upwind algorithm for computing turbulent flows on unstructured grids. Computers \&f Fluids 23, (1994), 1-21.

[3] F. P. T. Baaijens, An iterative solver for the DEVSS/DG method with application to smooth and non-smooth flows of the upper convected Maxwell fluid. J. Non-Newtonian Fluid Mech. 75, (1998), $119-138$.

[4] T. J. Barth, A 3-D upwind Euler solver for unstructured meshes. AIAA Paper 91-1548-CP, (1991).

[5] S. Balay, K. Buschelman, V. Eijkhout, W. D. Gropp, D. Kaushik, M. G. Knepley, L. C. McInnes, B. F. Smith and H. Zhang, PETSc User's Manual. ANL-95/11, Mathematics and Computer Science Division, Argonne National Laboratory, (2004). http://www-unix.mcs.anl.gov/petsc/petsc-2/

[6] M. Benzi, G. H. Golub and J. Liesen, Numerical solution of saddle point problems. Acta Numerica 14, (2005), 1-137.

[7] A. E. Caola, Y. L. Joo, R. C. Armstrong and R. A. Brown, Highly parallel time integration of viscoelastic flows. J. Non-Newtonian Fluid Mech. 100, (2001), 191-216.

[8] H.-S. Dou and N. Phan-Thien, Parallelisation of an unstructured finite volume code with PVM: viscoelastic flow around a cylinder. J. Non-Newtonian Fluid Mech. 77, (1998), 21-51.

[9] H. C. Elman, V. E. Howle, J. N. Shadid and R. S. Tuminaro, A parallel block multi-level preconditioner for the 3D incompressible Navier-Stokes equations. J. Comput. Phys. 187, (2003), 504-523.

[10] R. Falgout, A. Baker, E. Chow, V. E. Henson, E. Hill, J. Jones, T. Kolev, B. Lee, J. Painter, C. Tong, P. Vassilevski and U. M. Yang, User's manual, HYPRE High Performance Preconditioners. UCRLMA-137155 DR, Center for Applied Scientific Computing, Lawrence Livermore National Laboratory, (2002). http://www.llnl.gov/CASC/hypre/

[11] Y. R. Fan, R. I. Tanner and N. Phan-Thien, Galerkin/least-square finite element methods for steady viscoelastic flows. J. Non-Newtonian Fluid Mech. 84, (1999), 233-256.

[12] A. Fortin and M. Fortin, A preconditioned generalized minimum residual algorithm for the numerical solution of viscoelastic fluid flows. J. Non-Newtonian Fluid Mech. 36, (1990), 277-288.

[13] P. H. Gaskell and A. K. C. Lau, Curvature-compensated convective transport: SMART, an new boundedness-preserving transport algorithm. Int. J. Numer. Methods Fluids 8, (1988), 617-641.

[14] M. A. Hulsen, R. Fattal and R. Kupferman, Flow of viscoelastic fluids past a cylinder at high Weissenberg number: stabilized simulations using matrix logarithms. J. Non-Newtonian Fluid Mech. 127, (2005), 27-39.

[15] D. Hysom and A. Pothen, A scalable parallel algorithm for incomplete factor preconditioning. SIAM J. Sci. Comput. 22, (2001), 2194-2215.

[16] D. Kay, D. Loghin and A. J. Wathen, A preconditioner for the steady-state Navier-Stokes equations. SIAM J. Sci. Comput. 24, (2002), 237-256.

[17] J. M. Kim, C. Kim, K. H. Ahn and S. J. Lee, An efficient iterative solver and high-resolution computations of the Oldroyd-B fluid flow past a confined cylinder. J. Non-Newtonian Fluid Mech. 123, (2004), 161-173.

[18] B. P. Leonard, Stable and accurate convective modeling procedure based on quadratic upstream interpolation. Comp. Meth. Appl. Mech. Engrg 19, (1979), 59-98. 
[19] G. H. McKinley, R. C. Armstrong and R. A. Brown, The wake instability in viscoelastic flow past confined circular cylinders. Phil. Trans. R. Soc. Lond. A 344, (1993), 265-304.

[20] G. Mompean and M. Deville, Unsteady finite volume simulation of Oldroyd-B fluid through a threedimensional planar contraction. J. Non-Newtonian Fluid Mech. 72, (1997), 253-279.

[21] P. J. Oliveira, A. I. P. Miranda, A numerical study of steady and unsteady viscoelastic flow past bounded cylinders. J. Non-Newtonian Fluid Mech. 127, (2005), 51-66.

[22] P. J. Oliveira, F. T. Pinho and G. A. Pinto, Numerical simulation of non-linear elastic flows with a general collocated finite volume method. J. Non-Newtonian Fluid Mech. 79, (1998), 1-43.

[23] R. G. Owens, C. Chauvière and T. N. Phillips, A locally-upwinded spectral technique (LUST) for viscoelastic flows. J. Non-Newtonian Fluid Mech. 108, (2002), 49-71.

[24] C. M. Rhie and W. L. Chow, Numerical study of the turbulent flow past an airfoil with trailing edge separation. AIAA J. 21 (1983), 1525-1532.

[25] M. Rozloznik, Saddle point problems, iterative solution and preconditioning: a short review. Submitted to Proceedings of the XV-th Summer School Software and Algorithms of Numerical Mathematics, I. Marek ed., University of West Bohemia, Pilsen, (2003).

[26] Y. Saad and M. H. Schultz, GMRES: a generalized minimal residual algorithm for solving nonsymmetric linear systems, SIAM J. Sci. Statist. Comput. 7, (1986), 856-869.

[27] M. Sahin, A preconditioned semi-staggered dilation-free finite volume method for the incompressible Navier-Stokes equations on all-hexahedral elements. Int. J. Numer. Meth. Fluids 49, (2005), 959-974.

[28] G. P. Sasmal, A finite volume approach for calculation of viscoelastic flow through an abrupt axisymmetric contraction. Journal of Non-Newtonian Fluid Mechanics 56, (1995), 15-47.

[29] A. H. Shiang, A. Oztekin, J.-C. Lin and D. Rockwell, Hydroelastic instabilities in viscoelastic flow past a cylinder confined in a channel. Exp. Fluids 28, (2000), 128-142.

[30] M. D. Smith, Y. L. Joo, R. C. Armstrong and R. A. Brown, Linear stability analysis of flow of an Oldroyd-B fluid through a linear array of cylinders. J. Non-Newtonian Fluid Mech. 109, (2002), $13-50$.

[31] S.-C. Xue, N. Phan-Thien and R. I. Tanner, Three dimensional numerical simulations of viscoelastic flows through planar contractions. J. Non-Newtonian Fluid Mech. 72, (1998), 195-245.

[32] J. Y. Yoo and Y. Na, A numerical study of the planar contraction flow of a viscoelastic fluid using the SIMPLER algorithm. J. Non-Newtonian Fluid Mech. 39, (1991), 89-106.

[33] J. M. Verhelst and F. T. M. Nieuwstadt, Visco-elastic flow past circular cylinders in a channel: experimental measurement of velocity and drag. J. Non-Newtonian Fluid Mech. 116, (2004), 301328.

[34] P. Wapperom and M. F. Webster, A second-order hybrid finite-element/volume method for viscoelastic flows. J. Non-Newtonian Fluid Mech. 79, (1998), 405-431. 


\begin{tabular}{|c||c|c|c|c|c|c|}
\hline Mesh & Number of Nodes & Number of Elements & Total DOF & $\Delta r_{\min } / R$ & $\Delta S_{\min } / R$ & $\Delta S_{\max } / R$ \\
\hline \hline M1 & 6031 & 5824 & 35358 & 0.0133 & 0.0117 & 0.0785 \\
M2 & 21735 & 21320 & 128750 & 0.0062 & 0.0056 & 0.0392 \\
M3 & 70312 & 69519 & 418700 & 0.0031 & 0.0029 & 0.0196 \\
\hline
\end{tabular}

Table 1: Description of quadrilateral meshes used in the present work. $\Delta r_{\min }$ is the minimum normal mesh spacing and $\Delta S_{\min }$ and $\Delta S_{\max }$ are the minimum and maximum tangential mesh spacing on the cylinder surface.

\begin{tabular}{|c||c|c|c||c|c|c|}
\hline \multicolumn{1}{|c||}{} & \multicolumn{3}{c||}{$\begin{array}{c}\text { Maximum value of } T_{x x} \\
\text { next to cylinder wall }\end{array}$} & \multicolumn{3}{c|}{$\begin{array}{c}\text { Maximum value of } T_{x x} \\
\text { in the wake region }\end{array}$} \\
\hline$W e$ & M1 & M2 & M3 & M1 & M2 & M3 \\
\hline \hline 0.5 & 77.52 & 78.96 & 79.71 & 8.38 & 8.84 & 8.98 \\
0.6 & 91.72 & 92.97 & 93.73 & 14.26 & 16.08 & 16.97 \\
0.7 & 104.99 & 105.80 & 106.53 & 23.82 & 29.73 & 33.86 \\
0.8 & 116.88 & 116.96 & 117.78 & 38.67 & 55.06 & 70.42 \\
0.9 & 126.84 & 126.24 & 126.94 & 61.51 & 103.53 & 159.82 \\
\hline
\end{tabular}

Table 2: Convergence of maximum value of extra stress tensor component $T_{x x}$ at the cylinder wall and in the wake region with mesh refinement (Oldroyd-B fluid).

\begin{tabular}{|c||c|c|}
\hline Authors & $\begin{array}{c}\text { Maximum value of } T_{x x} \\
\text { at the cylinder wall }\end{array}$ & $\begin{array}{c}\text { Maximum value of } T_{x x} \\
\text { in the wake region }\end{array}$ \\
\hline \hline Present (M3) & 106.53 & 33.86 \\
Fan et al. [11] & 106.77 & 40.05 \\
Owens et al. $[23]$ & 106.4 & 37.1 \\
Alves et al. M60(WR) [1] & 100.02 & 38.33 \\
Kim et al. $[17]$ & 107.7 & 38.8 \\
\hline
\end{tabular}

Table 3: Comparison of maximum value of extra stress tensor component $T_{x x}$ at the cylinder wall and in the wake region at $W e=0.7$ (Oldroyd-B fluid). 


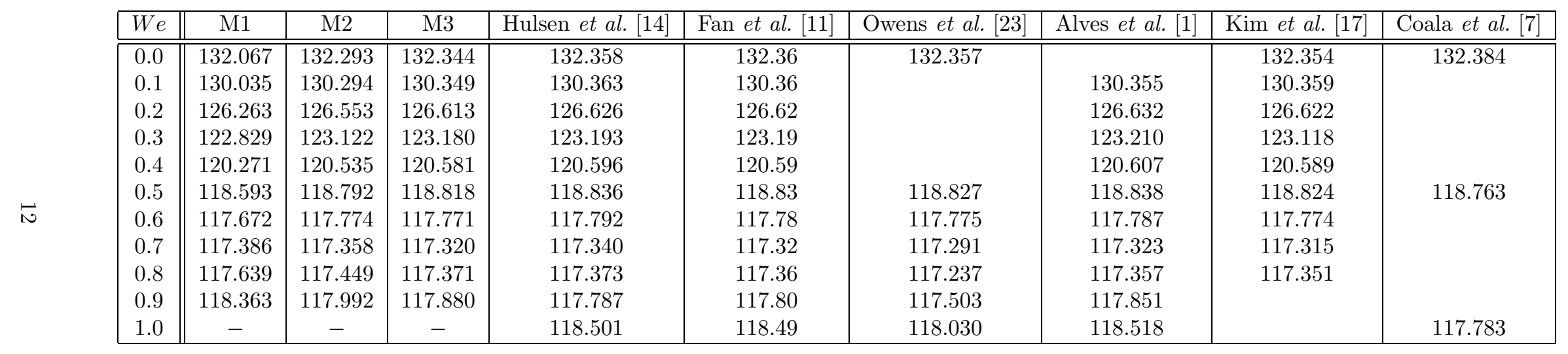

Table 4: Comparison of the dimensionless drag coefficient for 2D flow past a confined circular cylinder in a channel (Oldroyd-B fluid). 


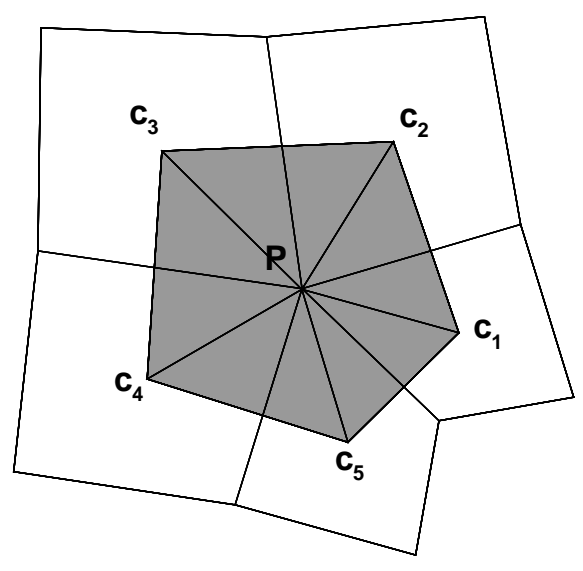

Figure 1: Two-dimensional unstructured mesh with a dual control volume surrounding a node $P$.

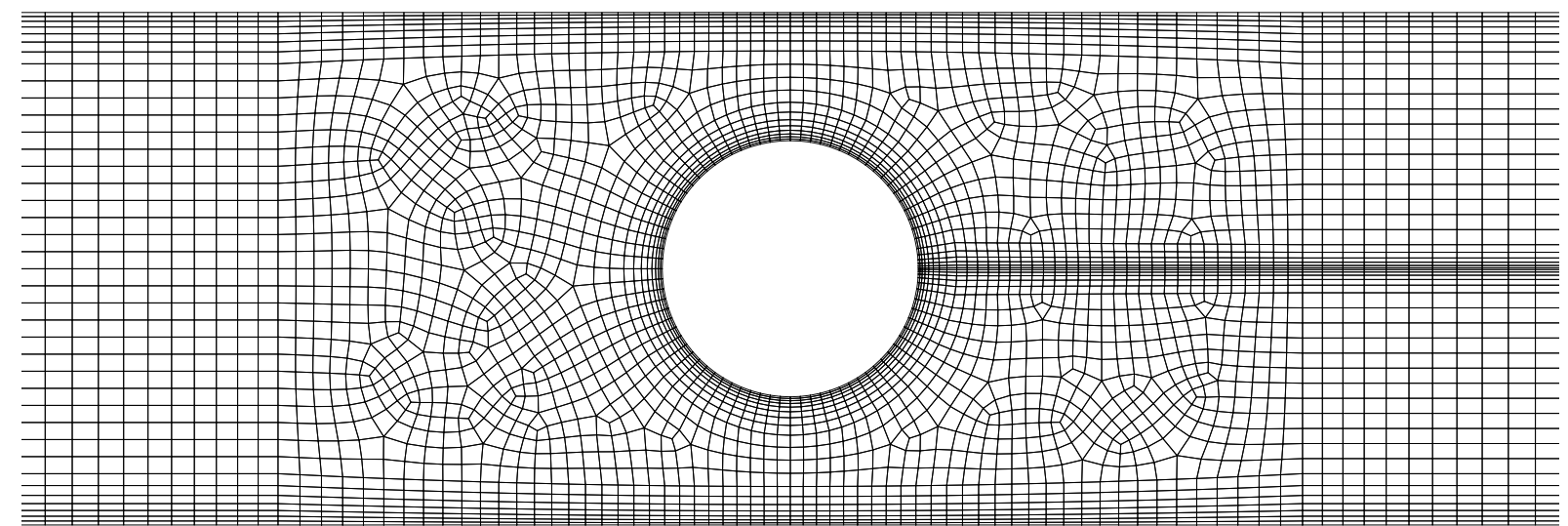

Figure 2: The computational coarse mesh M1 for the flow past a confined circular cylinder with 5824 elements and 6031 nodes. 


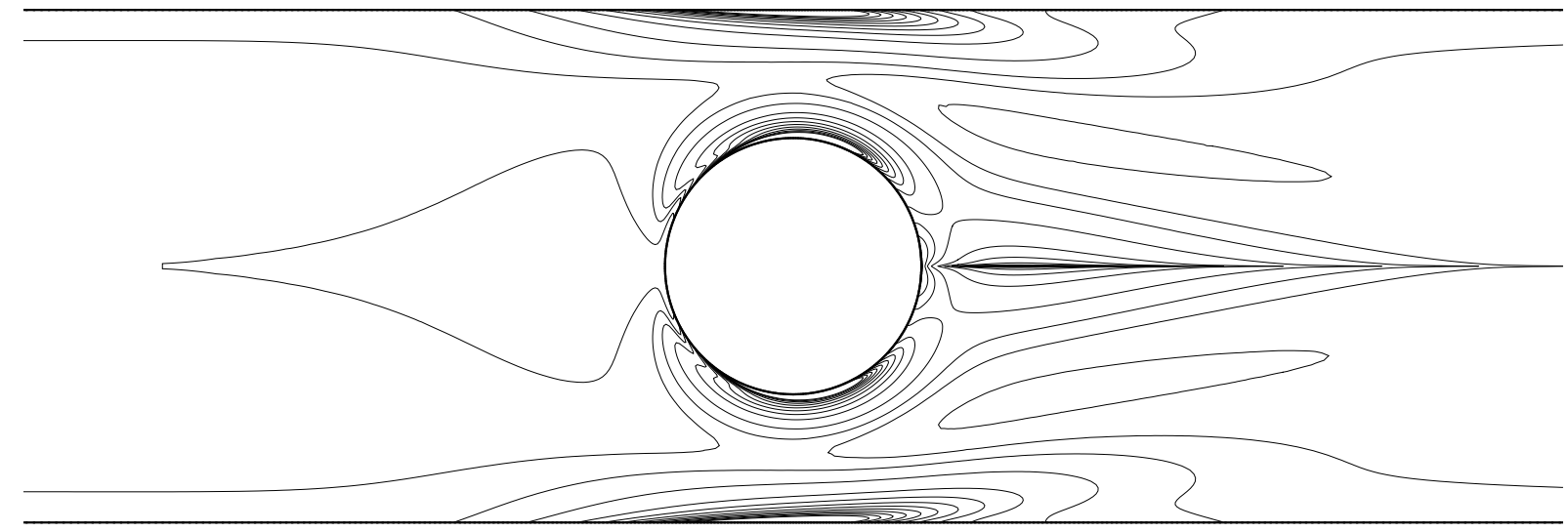

Figure 3: Computed steady state $T_{x x}$ contour plots at $W e=0.7$ on mesh M3 for an Oldroyd B fluid $(\beta=0.59)$. The contour levels shown are $0,1,2,4,8,12,16,20,24,28,32,36$ and 40.

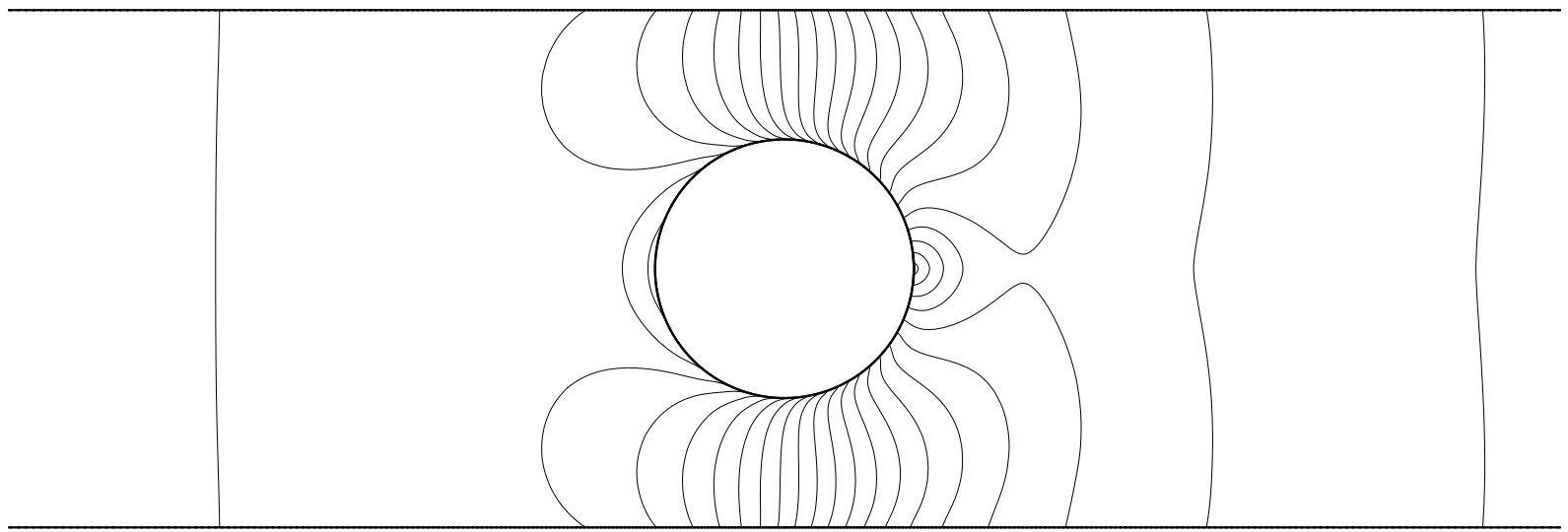

Figure 4: Computed steady state pressure contour plots at $W e=0.7$ on mesh M3 for an Oldroyd B fluid $(\beta=0.59)$. The difference between the contour levels is 2.5. The pressure field is smooth and free from checkerboard oscillations. 

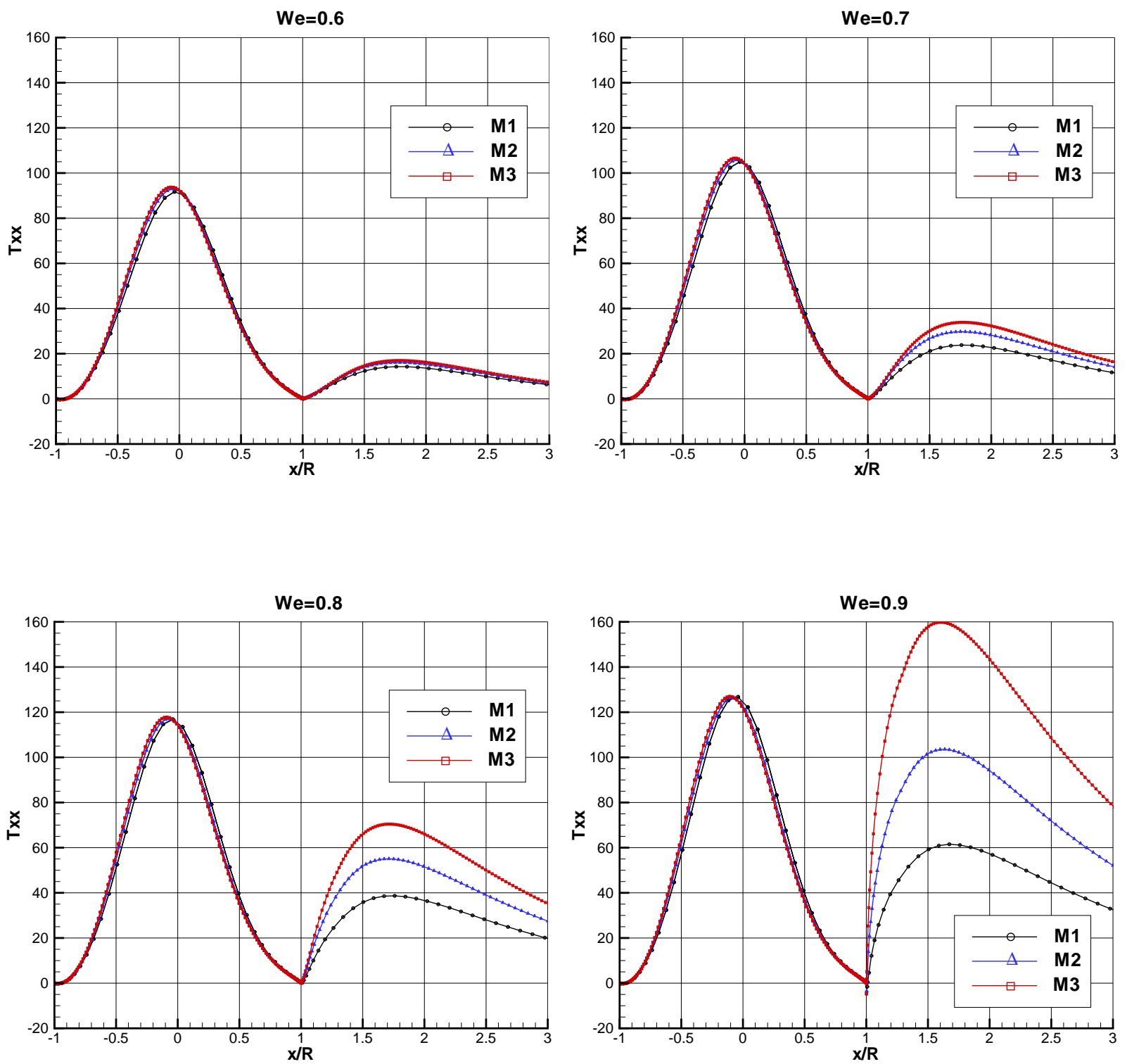

Figure 5: Convergence of $T_{x x}$ on the cylinder surface and in the cylinder wake for an Oldroyd B fluid $(\beta=0.59)$ at $W e=0.6,0.7,0.8$ and 0.9. Mesh convergence is observed up to $W e=0.7$; at $W e=0.9$ we can clearly see the effects of the high Weissenberg number problem. 


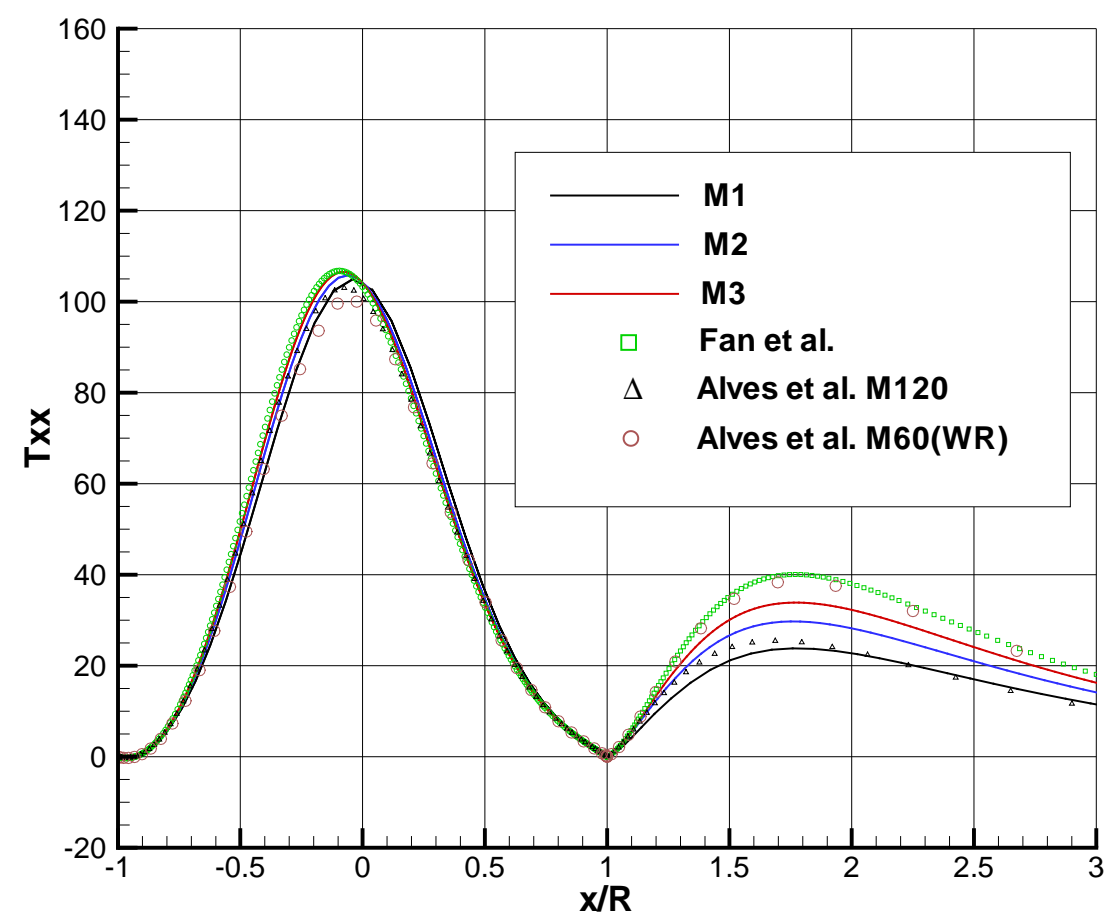

Figure 6: Comparison of $T_{x x}$ on the cylinder surface and in the cylinder wake at $W e=0.7$ for an Oldroyd B fluid $(\beta=0.59)$.

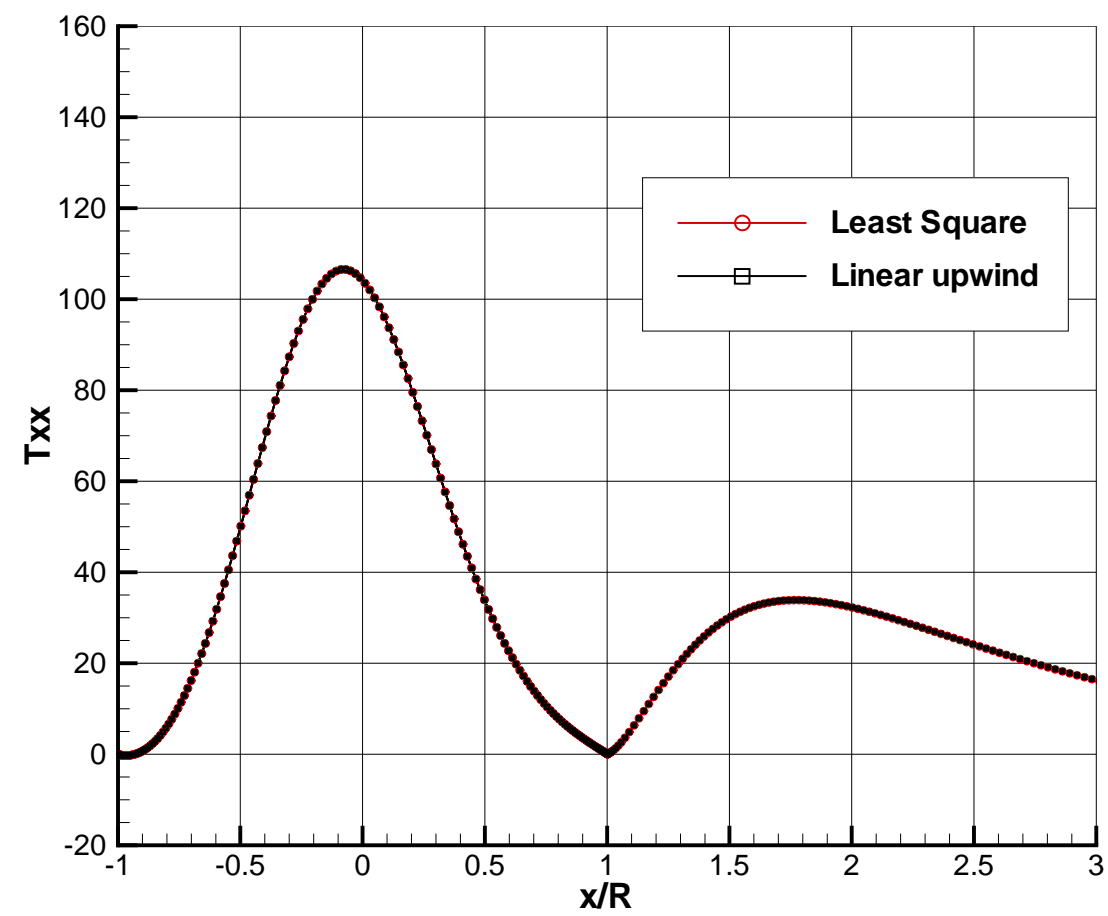

Figure 7: Comparison between least square and linear upwind interpolations for $T_{x x}$ on the cylinder surface and in the cylinder wake at $W e=0.7$ on mesh M3 for an Oldroyd B fluid $(\beta=0.59)$. 


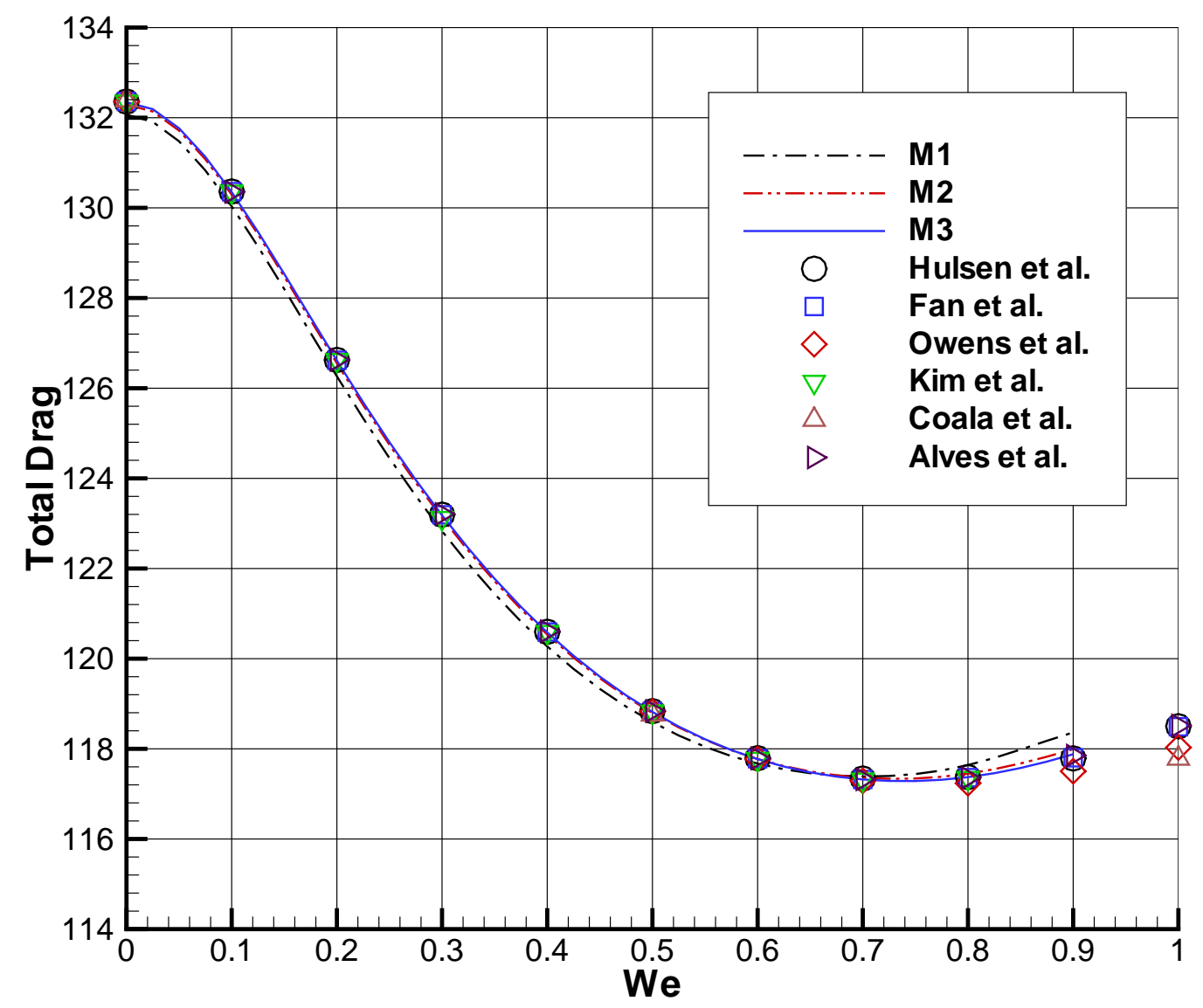

Figure 8: Comparison of steady state drag as a function of Weissenberg number for an Oldroyd B fluid $(\beta=0.59)$. 


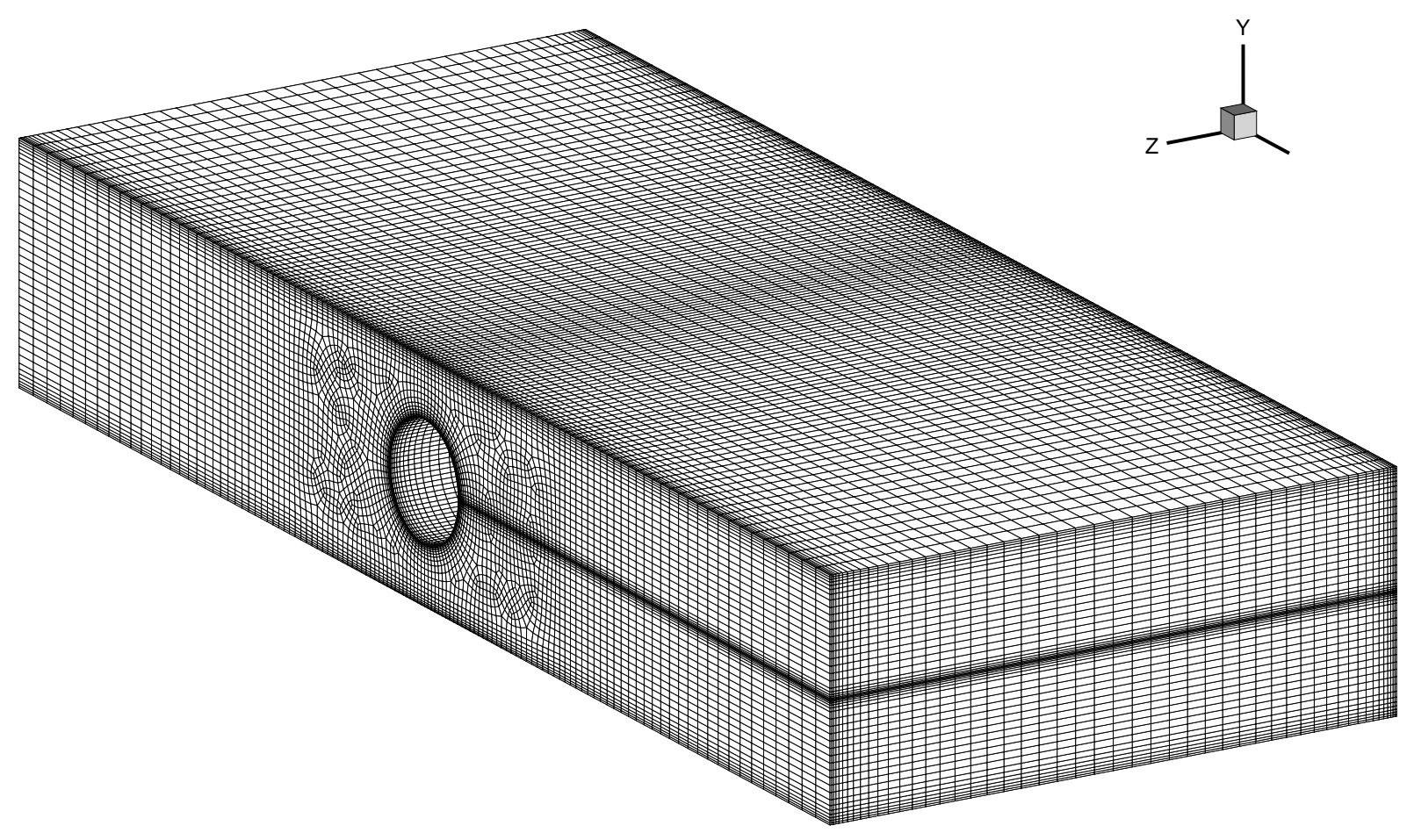

Figure 9: The computational coarse mesh for the flow past a confined circular cylinder in a rectangular channel with 307581 nodes and 291200 hexahedral elements $(R / H=0.5$ and $W / H=2.5)$. 

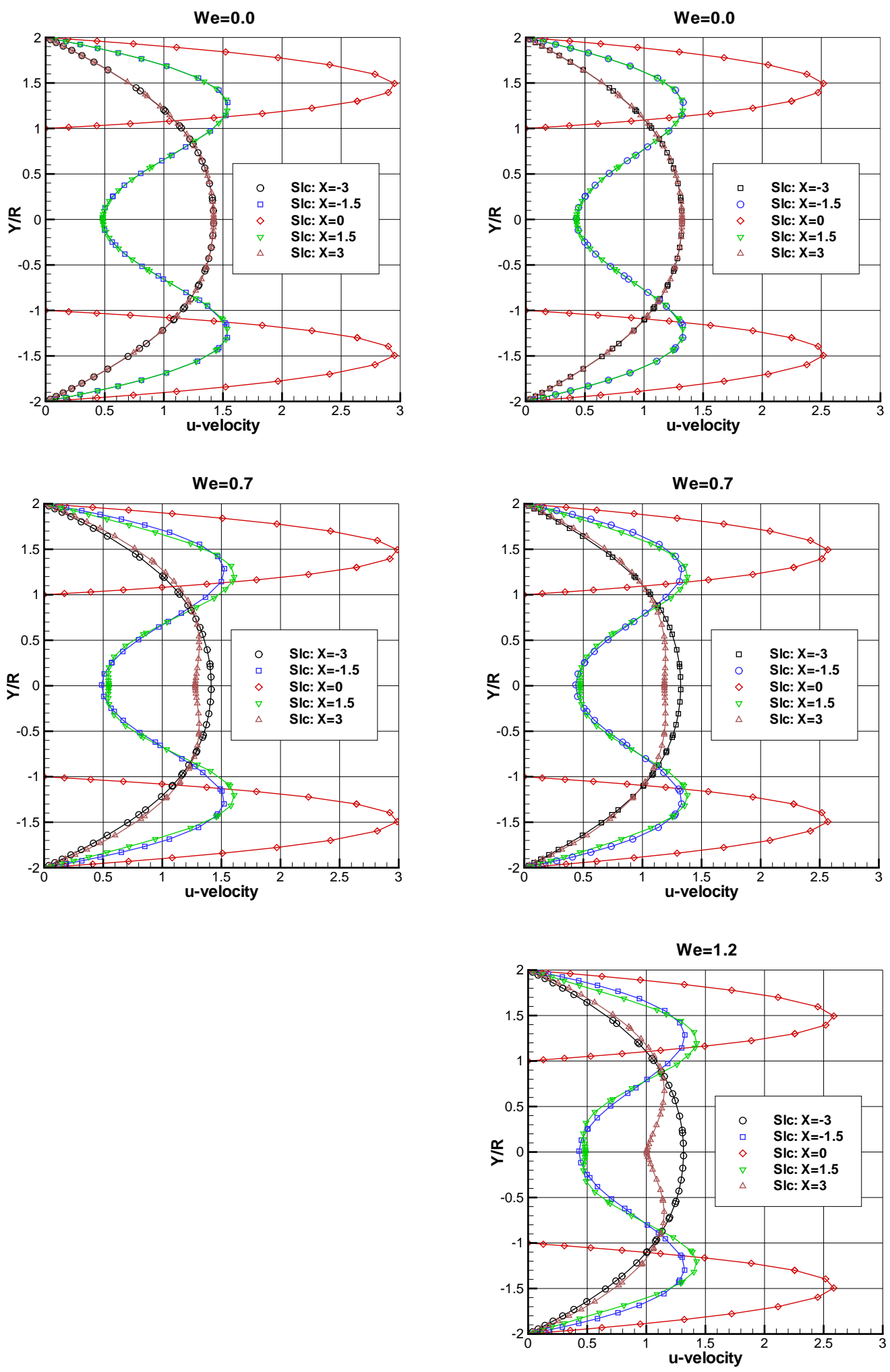

Figure 10: Comparison of the computed $x$-velocity component for 2D (left) and 3D (right) flow of an Oldroyd-B fluid past a confined circular cylinder in a rectangular channel $(\beta=0.59)$. 

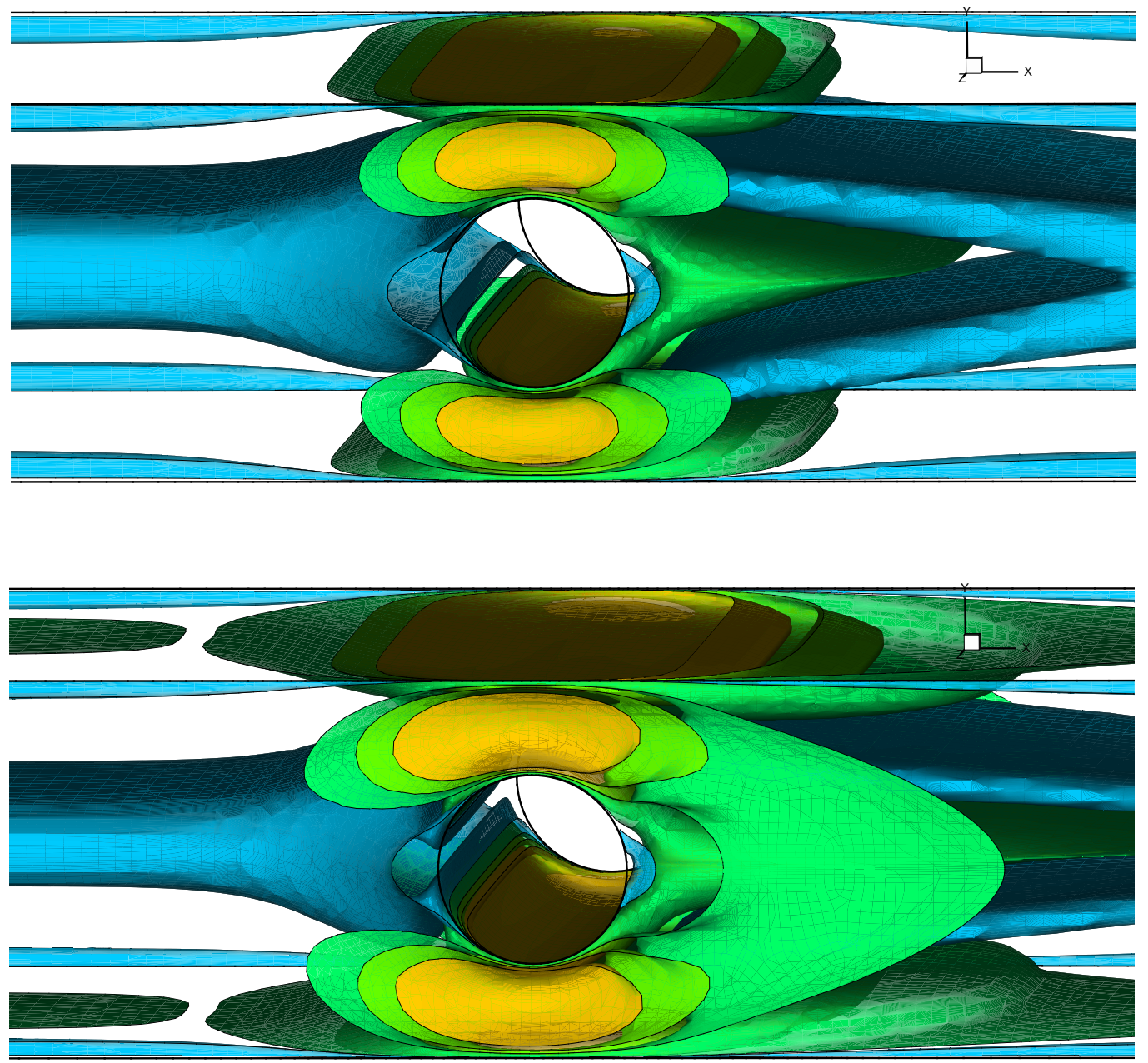

Figure 11: Computed 3D cell centre $T_{x x}$ contours at $W e=0.7$ (upper) and $W e=1.2$ (lower) for flow of an Oldroyd-B fluid past a confined circular cylinder in a rectangular channel $(\beta=0.59)$. The contour levels shown for each plot are $0.1,2,4$ and 8 . On the side walls the closed contours enclose region of high stresses. 

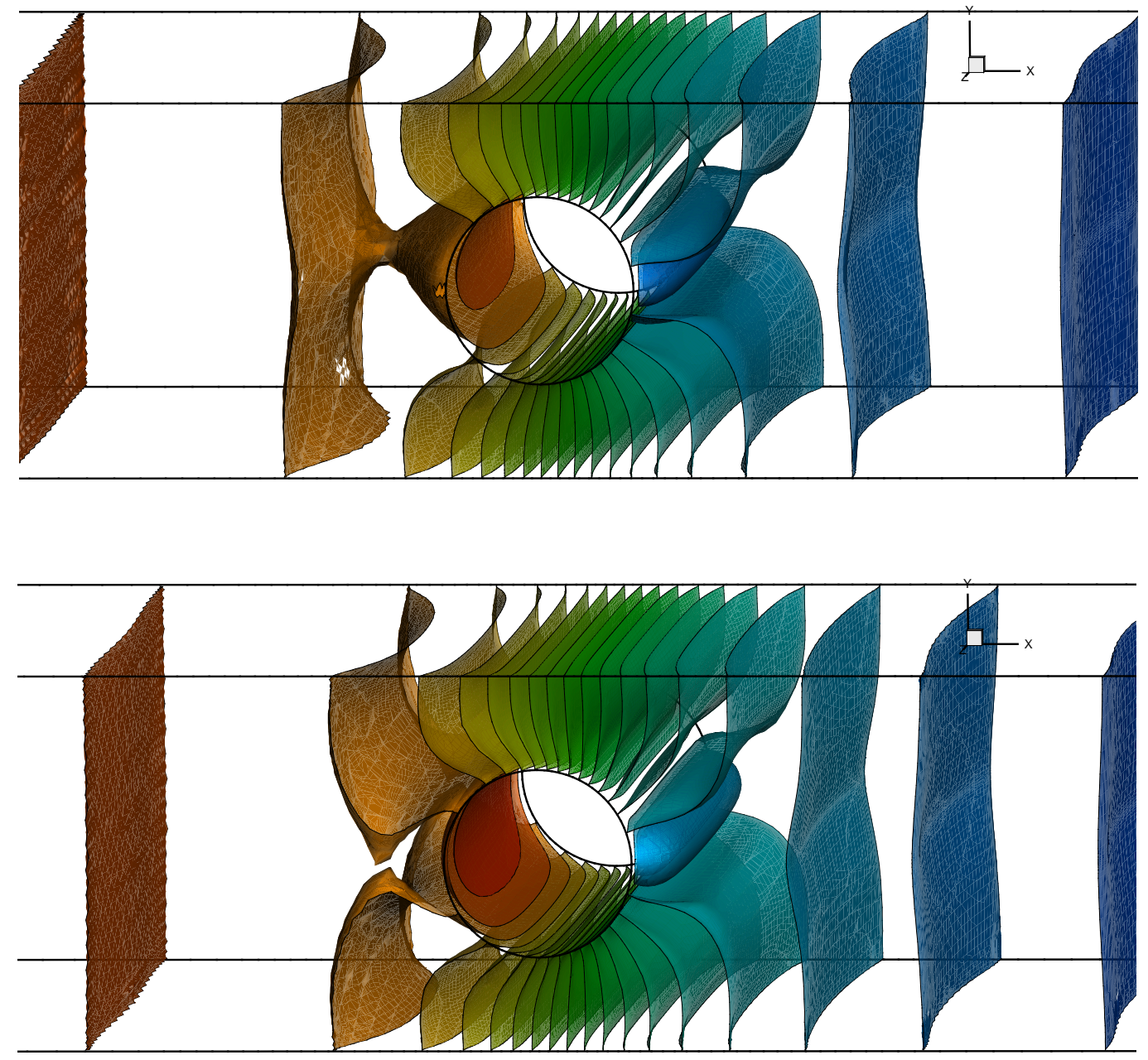

Figure 12: Computed 3D cell centre pressure contours at $W e=0.7$ (upper) and $W e=1.2$ (lower) for flow of an Oldroyd-B fluid past a confined circular cylinder in a rectangular channel $(\beta=0.59)$. The difference between the pressure contour levels is 2.5 . 


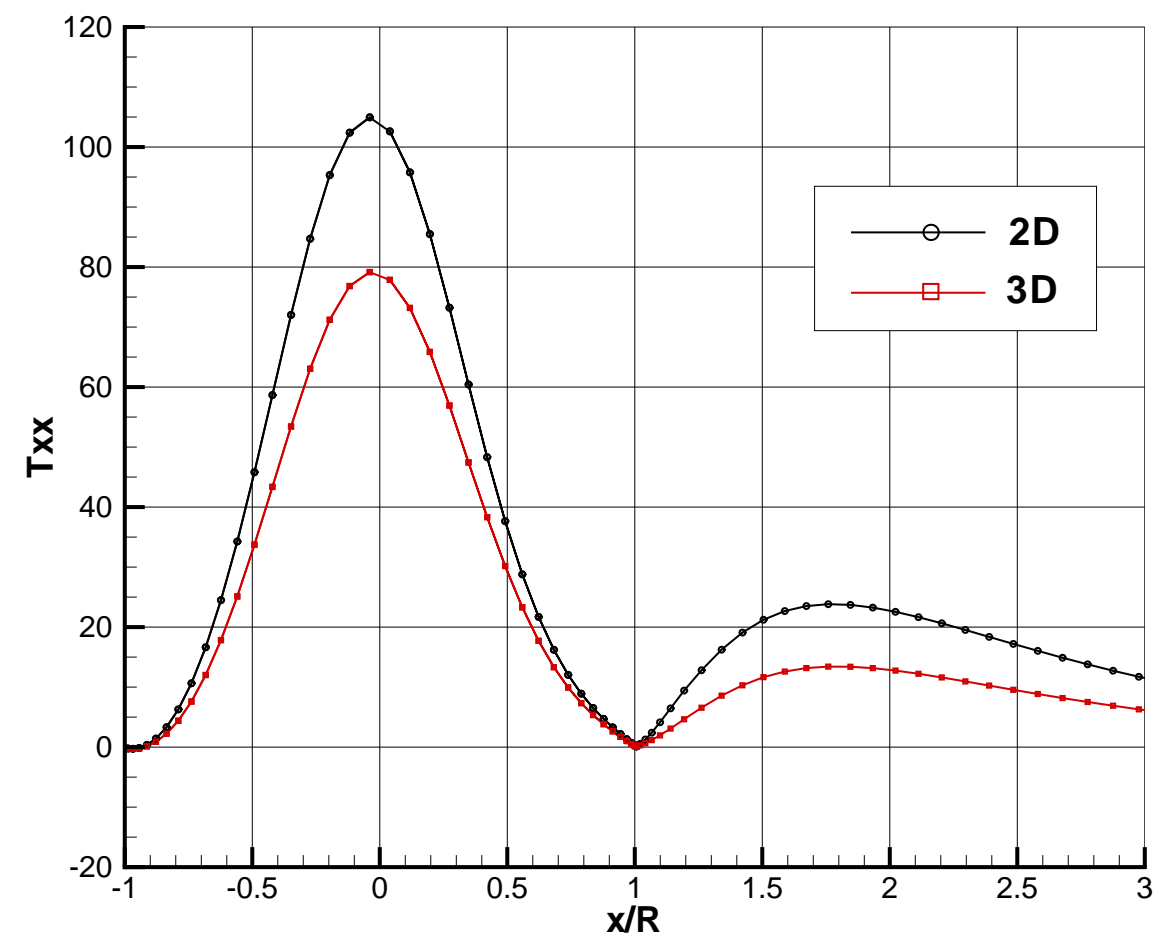

Figure 13: Comparison of $2 \mathrm{D}$ and $3 \mathrm{D} T_{x x}$ on the cylinder surface and in the cylinder wake on the $z=0$ symmetry plane for an Oldroyd B fluid at $W e=0.7(\beta=0.59)$. 


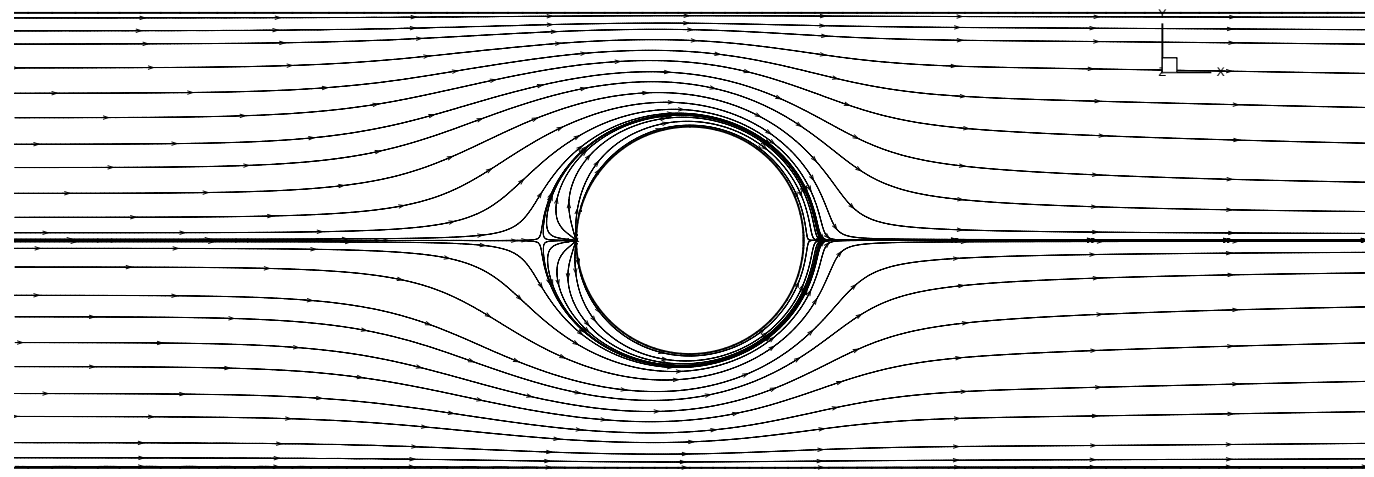

Figure 14: Computed 2D streamtraces close to the side plates at $z= \pm 4.99 R$ for flow of an Oldroyd-B fluid past a confined circular cylinder in a rectangular channel at $W e=2.0(\beta=0.59)$. Transient, just before onset of oscillations due to the HWNP.

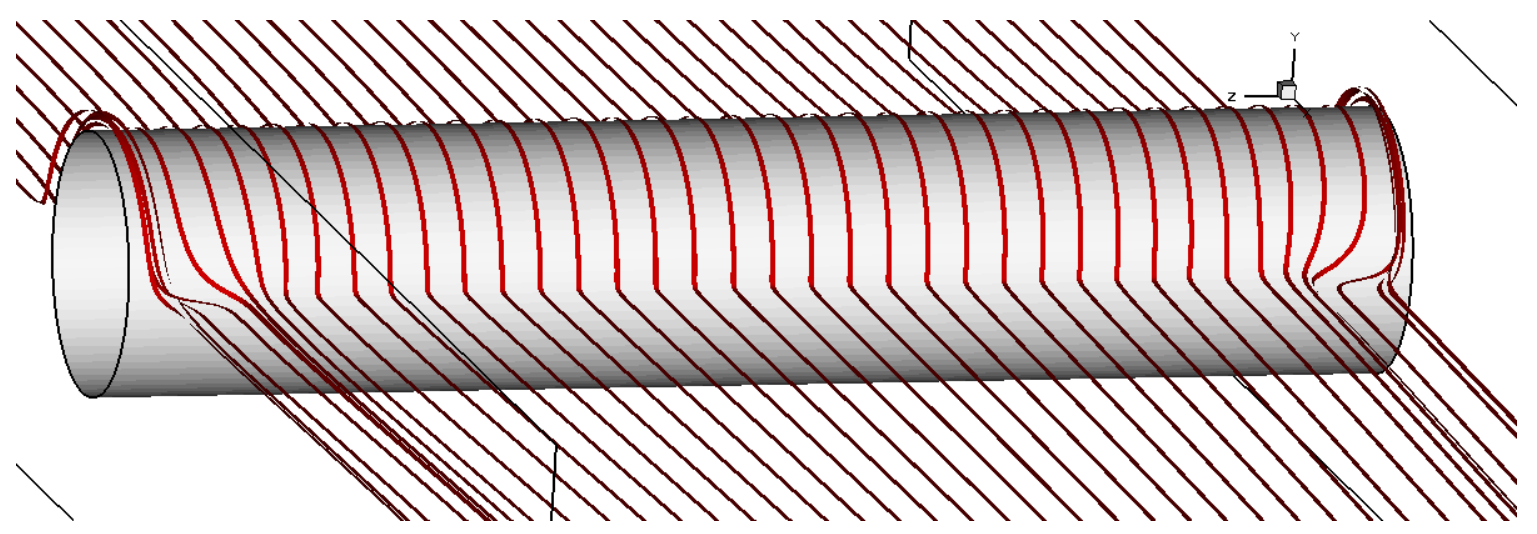

Figure 15: Computed streamtrace plot at $W e=2.0$ for flow of an Oldroyd-B fluid past a confined circular cylinder in a rectangular channel $(\beta=0.59)$. Transient, just before HWNP. 